\title{
Xp22.33p22.12 Duplication in a Patient with Intellectual Disability and Dysmorphic Facial Features
}

\author{
Carla Lintas ${ }^{a, b}$ Chiara Picinellic Ignazio S. Piras ${ }^{c}$ Roberto Sacco ${ }^{a, b}$ \\ Stefano Gabriele ${ }^{b}$ Magda Verdecchia ${ }^{a}$ Antonio M. Persico ${ }^{a-c}$ \\ a Unit of Child and Adolescent NeuroPsychiatry and b ${ }^{\mathrm{L}}$ aboratory of Molecular Psychiatry and Neurogenetics, \\ Department of Medicine, University Campus Bio-Medico, Rome, and ' Mafalda Luce Center for Pervasive \\ Developmental Disorders, Milan, Italy
}

\section{Key Words}

Duplication Xp22.33p22.12 - Dysmorphic facial features . Intellectual disability

\begin{abstract}
Anovel 19.98-Mb duplication in chromosomeXp22.33p22.12 was detected by array CGH in a 30-year-old man affected by intellectual disability, congenital hypotonia and dysmorphic features. The duplication encompasses more than 100 known genes. Many of these genes (such as neuroligin 4, cyclin-dependent kinase like 5, and others) have already correlated with $\mathrm{X}$-linked intellectual disability and/or neurodevelopmental disorders. Due to the high number of potentially pathogenic genes involved in the reported duplication, we cannot correlate the clinical phenotype to a single gene. Indeed, we suggest that the resulting clinical phenotype may have arisen from the overexpression and consequent perturbation of fine gene dosage.

๑) 2016 S. Karger AG, Basel
\end{abstract}

X-linked mental retardation is a common clinically complex and genetically heterogeneous condition arising from mutations and/or copy number variants in many genes located in the $\mathrm{X}$ chromosome [Ropers et al., 2005]. Xp duplications have been described in males affected by intellectual disability and variable congenital abnormalities [Honda et al., 2010; Salaria et al., 2012; Wu et al., 2013].

We hereby report on a 30-year-old man with a de novo 19.98-Mb duplication in chromosome Xp22.33p22.12 affected by severe intellectual disability, congenital hypotonia and dysmorphic features, including prominent glabella, short philtrum, micrognathia, high forehead, hypertelorism, epicanthal folds, downslanting palpebral fissures, wide nasal bridge, and short upper lip.

\section{Case Report}

The proband, an adult male now 30 years old, was born at 38 weeks of gestation by spontaneous delivery from nonconsanguineous and healthy parents. Prenatal data are not available. His birth weight was normal being $3,300 \mathrm{~kg}$ (50th centile). No intrauterine or postnatal growth delay was recorded. At 6 months, hypotonia and microcephaly with craniostenosis were diagnosed, but at 4 years of age, cranial growth had partly recovered, and his head circumference was normal by the age of 9 . Bilateral cryptorchidism was corrected surgically at 3 years of age. Physical examination identified dysmorphic features, including prominent glabella, short philtrum, micrognathia, high forehead, hypertelorism,

\section{KARGER 125}

(c) 2016 S. Karger AG, Base

$1661-8769 / 16 / 0065-0236 \$ 39.50 / 0$

E-Mail karger@karger.com

www.karger.com/msy
Antonio M. Persico

Unit of Child and Adolescent NeuroPsychiatry, Department of Medicine

University Campus Bio-Medico

Via Alvato del Portillo 21, IT-00128 Rome (Italy)

E-Mail a.persico@unicampus.it 
Fig. 1. The patient at 30 years old. a Skeletal features include thin legs, right radioulnar synostosis, valgus deformity, and talipes equinovarus. b Dismorphic facial features include prominent glabella, short philtrum, high forehead, hypertelorism, epicanthal folds, downslanting palpebral fissures, wide nasal bridge, and short upper lip.

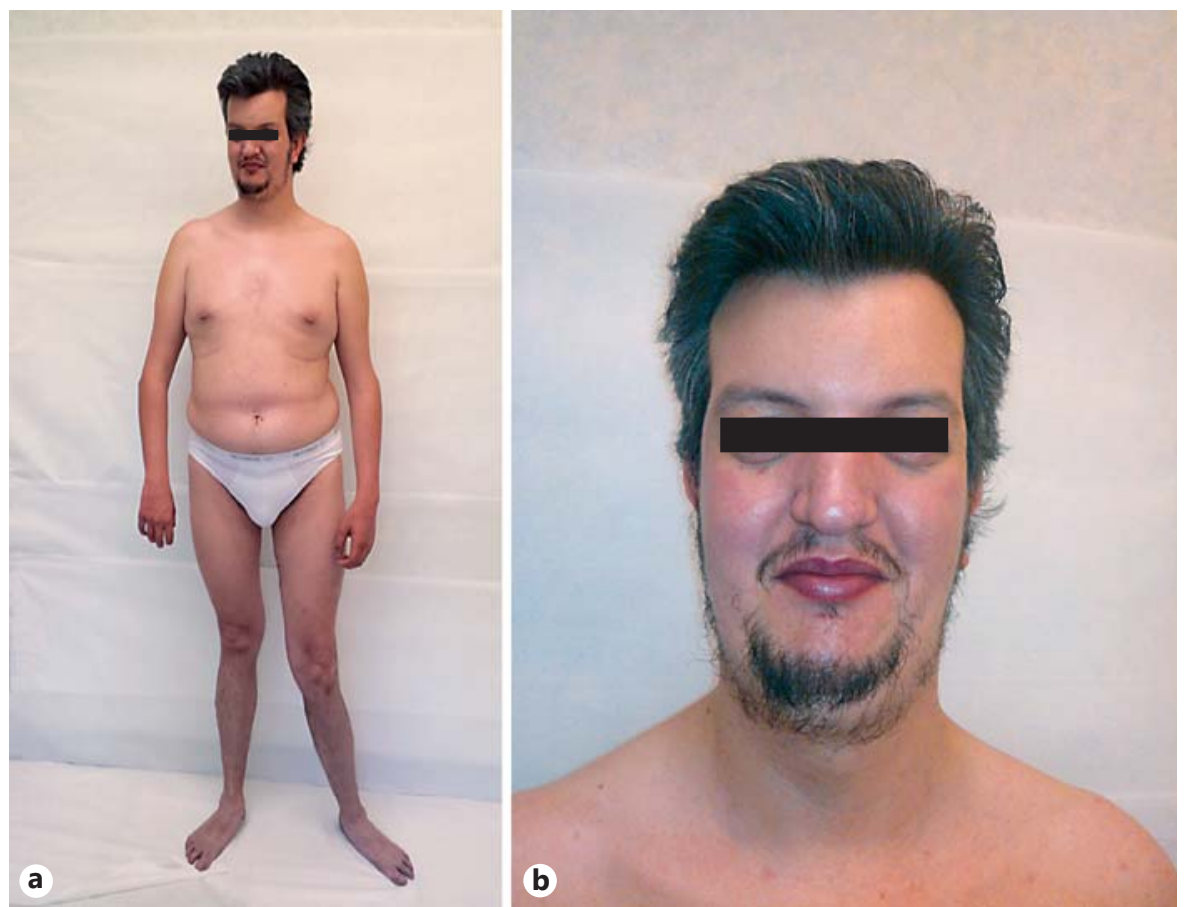

epicanthal folds, downslanting palpebral fissures, wide nasal bridge, ogival palate, short upper lip, and hypodontia (fig. 1). Some, though not all developmental milestones were delayed: he walked unsupported at 24 months, but expressive language for single words began at about 16 months. Hearing acuity was found throughout his life and was tested approximately every 2 years. Mild visual anomalies were present including astigmatism and hyperopia diagnosed at age 5 and 13, respectively. Cardiac and abdominal sonograms were normal during childhood, but by 17 years of age he developed a mitral and tricuspid valve prolapse as well as prominent varicose veins. His bone age was normal at 6 years, but scoliosis developed during adolescence. Other skeletal features include thin legs, right radioulnar synostosis, valgus deformity, and talipes equinovarus (fig. 1). The patient's nail growth is absent, tooth eruption was delayed and some permanent teeth never erupted. Classical G-banding, EEG, MRI, and CT scans were all normal, reporting only a slight asymmetry of the lateral ventricles. Neurological and neuropsychological deficits currently include severe intellectual disability (Coloured Progressive Matrices score: $17 / 36,<10$ th percentile), attention deficit/hyperactivity disorder with only attention deficits and no hyperactivity, verbal and cognitive perseverative behavior, ideomotor apraxia with difficulty in recognizing and imitating symbolic gestures, poor visuomotor integration and bimanual coordination, severe oropharyngeal dyspraxia with phonological deficits and swallowing disorders, hypostenia, and hypereflexia. Additional deficits include (a) graphic perseveration and/or impairment in spatial abilities with visuoconstructional apraxia (Rey complex figure score: $4 / 36$; <10th percentile), (b) visual executive functions (clockdrawing test and trail-making test), and (c) phonological fluency (no words produced for 3 phonemic cues in the controlled oral word association test) and naming pictured objects (Boston naming test score: $34 / 60$ ). Functions found within the normal range, following the correction for age and education level include the recognition of incomplete and fragmented figures (Street test: 7/14) and recalling a list of words when tested by an additional recognition trial (Rey auditory verbal learning scores for immediate recall $=14.1 / 75$; for delayed recall $=3.4 / 15$; for recognition $=$ $12 / 15)$. The patient's better recognition score compared to the immediate and delayed recall scores are compatible with a specific deficit in information retrieval, while information storage is relatively spared. Since his childhood, the patient underwent consecutive periods of rehabilitation, especially speech therapy. This may have contributed to the production of words semantically correlated with the target picture and of gestures appropriated for the use of an object. His parents also reported a specific ability in learning the names of some word categories (e.g. car models). Periodic reports from the rehabilitation team retrospectively document a slow, but constant improvement over time in many areas including social, communicative, motor, and language skills. Despite this improvement, severe intellectual disability and dysmorphic features persisted into adulthood. As an adult, he is interested in music, photography, voluntary work, and singing. $\mathrm{He}$ likes to help in simple household jobs, has a friendly attitude and makes contact easily. As a child, he was diagnosed with a mild form of Wolf-Hirschhorn syndrome (WHS) without a typical WHS 4p16.3 deletion [Zollino et al., 1996] and without intrauterine and postnatal body growth delay which is a core feature of WHS [Moretti et al., 2001]. Indeed, the facial resemblance between our patient and patients with classical WHS are no longer pronounced, suggesting that this patient had a developmental trajectory bringing him outside of the WHS phenotype. 


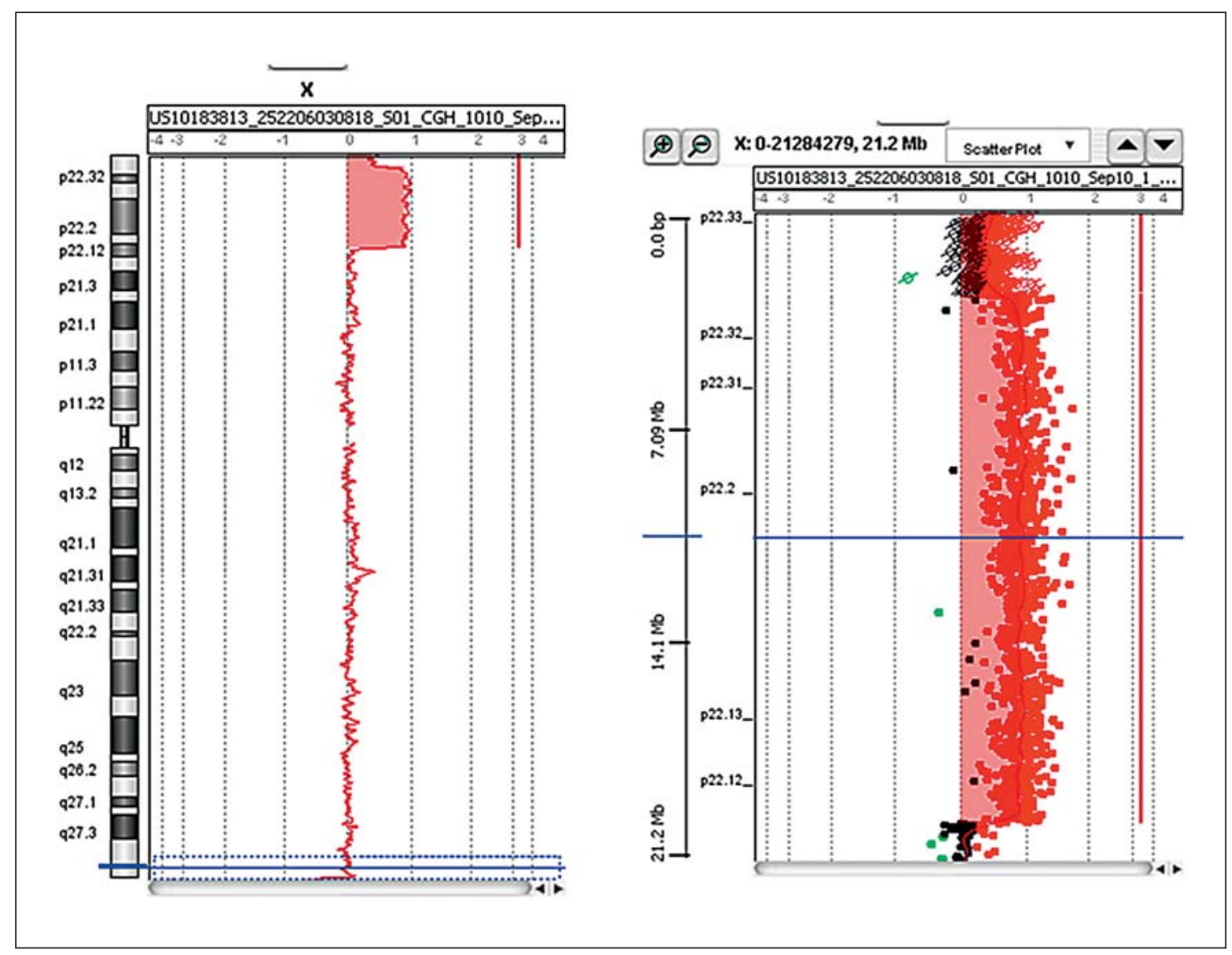

Fig. 2. The 20-Mb duplication in chromosome band Xp22.33p22.12 between 61,091 and 20,038,601 bp (Build 37: February 2009) detected using SurePrint G3 Human CGH 4x180K Microarray kit.

\section{Methods}

Array CGH was performed using the SurePrint G3 Human CGH 4x180K Microarray kit (Agilent Technologies, Santa Clara, Calif., USA) on genomic DNA from blood. The duplication was confirmed using a TaqMan Copy Number Assay targeting intron 3 of the retinoblastoma-binding protein 7 (RBBP7) gene and normalized with the RNase $P$ gene. RNA was extracted from peripheral blood mononuclear cells, retrotranscribed into cDNA and used to quantify RBBP7 mRNA level by TaqMan methodology; expression values were normalized to the housekeeping gene glyceraldehyde 3-phosphate dehydrogenase (GAPDH), used as an internal control. Network analysis was performed using the web tool 'STRING' 9.1 [Franceschini et al., 2013].

\section{Results and Discussion}

The patient is carrier of a de novo duplication located in chromosome Xp22.33p22.12, spanning $19.98 \mathrm{Mb}$ and encompassing more than 100 genes (fig. 2; table 1). The duplicated region started from oligomer A_18_P17041097 $(61,091 \mathrm{bp}$, first deleted) to oligomer A_16_P03666848 $(20,038,601 \mathrm{bp}$, last deleted). A total of 1,856 gains and 54 losses have been reported in this region in the Database of Genomic Variants [MacDonald et al., 2013], each overlapping well below $10 \%$ with the present aberration. The $\mathrm{Xp}$ duplication harbors several genes classified as 'disease genes' in the OMIM database of genetic disorders (http:// www.omim.org/). Single nucleotide mutations and/or deletions in some of these genes have been associated with neurodevelopmental disorders including neuroligin 4 (NLGN4; OMIM 300427, associated with autism, intellectual disability, Asperger syndrome), cyclin-dependent kinase like 5 (CDKL5; OMIM 300203, associated with Rett syndrome, epileptic encephalopathy), orofacial digital syndrome I (OFD1; OMIM 300170, associated with Joubert syndrome characterized by hypotonia, cerebellar ataxia and developmental delay), and adaptor-related protein complex 1 sigma 2 subunit (AP1S2; OMIM 
Table 1. Comparison of clinical features observed in our patient and in patients with overlapping Xp22.31p22.12 duplication

\begin{tabular}{llll}
\hline Locus Size & Ghenotype & Geference
\end{tabular}

Xp22.33p22.12 $19.98 \mathrm{Mb}$ intellectual disability, congenital hypotonia, dysmorphic features

PLCXD1, GTPBP6, NCRNA00107, PPP2R3B, SHOX,CRLF2,

present case CSF2RA,IL3RA, SLC25A6, NCRNA00105, ASMTL, P2RY8, SFRS17A, ASMT, DHRSX,ZBED1,CD99, XG, XGPY2, GYG2, ARSD, ARSE, ARSH, ARSF, MXRA5, PRKX, NLGN4X, VCXA, HDHD1A, STS, VCX, PNPLA4, MIR651, VCX2, VCX3B, KAL1, FAM9A, FAM9B, TBL1X, GPR143, SHROOM2, WWC3, CLCN4, MID1, HCCS, ARHGAP6, AMELX, MSL3, FRMPD4, PRPS2, TLR7, LOC349408, TLR8, TMSL3, TMSB4X, FAM9C, ATXN3L, EGFL6, TCEANC, RAB9A, TRAPPC2, OFD1, GPM6B, GEMIN8, GLRA2, FANCB, MOSPD2, ASB9, ASB11, PIGA, FIGF, PIR, BMX, ACE2, TMEM27, CA5BP, CA5B, INE2, ZRSR2, AP1S2, GRPR, CTPS2, S100G, SYAP1, CXorf15, RBBP7, REPS2, NHS, SCML1, RAI2, BEND2, SCML2, CDKL5, RS1, PPEF1, LOC100132163, PHKA2, GPR64, PDHA1, MAP3K15, SH3KBP1, CXorf23, LOC729609, MAP7D2

\begin{tabular}{|c|c|c|c|c|}
\hline $\mathrm{Xp} 22.31 \mathrm{p} 22.2$ & $2.8 \mathrm{Mb}$ & $\begin{array}{l}\text { ichthyosis, congenital heart defect, } \\
\text { varicose veins, speech delay, } \\
\text { hypogonadotropic hypogonadism }\end{array}$ & $\begin{array}{l}\text { STS, VCX, PNPLA4, MIR651, VCX2, VCX3B, KAL1, FAM9A, } \\
\text { FAM9B, TBL1X, GPR143, SHROOM2, WWC3 }\end{array}$ & Salaria et al., 2012 \\
\hline $\mathrm{Xp} 22.2$ & $737 \mathrm{~kb}$ & $\begin{array}{l}\text { West syndrome, severe mental } \\
\text { retardation, epilepsy, absent speech }\end{array}$ & REPS2, NHS & Honda et al., 2010 \\
\hline $\mathrm{Xp} 22.2$ & $737 \mathrm{~kb}$ & $\begin{array}{l}\text { moderate mental retardation, } \\
\text { speech delay, autistic features }\end{array}$ & REPS2, NHS & Honda et al., 2010 \\
\hline Xp22.33 & $290 \mathrm{~kb}$ & intellectual disability & SHOX & DECIPHER, 262464 \\
\hline Xp22.33 & $982 \mathrm{~kb}$ & global developmental delay & SHOX, CRLF2, CSF2RA, IL3RA & DECIPHER, 294106 \\
\hline $\mathrm{Xp} 22.33 \mathrm{p} 22.32$ & $2.2 \mathrm{Mb}$ & autism & $\begin{array}{l}\text { AK000470, AK000470, AK097803, ARSD, ARSE, ARSF, } \\
\text { ARSH, CXorf28, GYG2, LOC389906, MXRA5, PRKX, XG }\end{array}$ & DECIPHER, 294320 \\
\hline Xp22.31 & $1.57 \mathrm{Mb}$ & $\begin{array}{l}\text { global developmental delay, } \\
\text { abnormal facial shape }\end{array}$ & HDHD1A, STS, VCX, PNPLA4, MIR651 & DECIPHER, 280977 \\
\hline Xp22.33 & $1.5 \mathrm{Mb}$ & intellectual disability & HDHD1A, STS, VCX, PNPLA4, MIR651 & DECIPHER, 251816 \\
\hline
\end{tabular}

300629, associated with intellectual disability). Other OMIM genes correlated with known disorders, but not directly to neurodevelopment disorders, include short stature homeobox (SHOX; OMIM 312865, associated with Langer mesomelic dysplasia), steroid sulfatase (STS; OMIM 308100, associated with ichthyosis), arysulfatase E (ARSE; OMIM 300180, associated with chondrodysplasia punctate), Kallmann syndrome interval gene 1 (KAL1; OMIM 300836, associated with hypogonadotropic hypogonadism), midline 1 (M1D1; OMIM 300552, associated with Opitz GBBB syndrome type I), amelogenin (AMELX; OMIM 300391, associated with amelogenesis imperfecta type 1E), Nance-Horan syndrome gene (NHS; OMIM
300457, associated with cataract), retinoschisin ( $R S 1$; OMIM 300839, associated with retinoschisis), phosphorylase kinase liver alpha-2 subunit (PHKA2; OMIM 300798 , associated with glycogen storage disease), and pyruvate dehydrogenase alpha-1 (PDHA1; OMIM 300502, associated with pyruvate dehydrogenase E1-alphal deficiency). In addition, many other genes within the duplication play an important role in the nervous system and have been found to be associated with neurodevelopmental disorders. The ferm and PDZ domains containing the protein 4 gene (FRMPD4), a positive regulator of dendritic spine morphogenesis and density, has been found mutated in patients affected by intellectual disabil- 
ity [Rauch et al., 2012], autism and schizophrenia [Piton et al., 2011]. Similarly, de novo mutations in the neuronal synapse-associated protein 1 gene (SYAP1) and in the glycine receptor alpha-2 subunit gene (GLRA2), a ligandgated channel mediating a chloride-dependent inhibitory neurotransmission, have been found, respectively, in patients with autistic disorder [Prasad et al., 2012] and in patients affected by autism and schizophrenia [Piton et al., 2010]. A search for other patients with an Xp22.33p22.12-duplicated chromosome in the literature and in the Database of Chromosomal Imbalance and Phenotype in Humans DECIPHER (http://decipher. sanger.ac.uk) revealed many patients with even much smaller duplications in this chromosome location (table 1). The DECIPHER database reports $\sim 300$ patients with overlapping duplications ranging in size from $2 \mathrm{~kb}$ to $155 \mathrm{Mb}$. However, many of these patients, especially those with larger duplications, harbor additional chromosomal imbalances in their genome and, therefore, cannot be directly compared to our patient. Table 1 summarizes patients carrying unique Xp22.33p22.12 rearrangements. All DECIPHER patients (262464, 264106, 294320 , 280977, 289637, and 251816) have intellectual disability and/or neurodevelopmental disorders including autism, delayed speech or language development and dysmorphic features. The patient described by Salaria et al. [2012] harbors a 2.8-Mb Xp22.31p22.2 duplication in his genome: he had speech delay, ichthyosis, congenital heart defects, hypogonadotropic hypogonadism, varicose veins, and hypotonia. The 2 latter anomalies were also present in our patient. Patients with an Xp22.2 duplication [Honda et al., 2010] have mental retardation ranging from mild to severe and/or autistic traits and epilepsy (table 1).

Although many of the dysmorphic features presented in our case are relatively nonspecific, a mild form of WHS was clinically diagnosed as a child [Zollino et al., 1996]. This initial diagnosis questions whether any gene spanned by the present duplication may functionally interact with the Wolf-Hirschhorn candidate gene 1 (WHSC1) located in chromosome 4p16.3, so as to possibly explain the initial WHS-like features with a relatively favorable developmental trajectory. According to network analysis, the only interaction between any duplicated gene and WHSC1 involves $R B B P 7$, which encodes for the retinoblastoma binding proten 7 , a transcriptional regulator. STRING [Franceschini et al., 2013] detects protein-protein interactions supported by experimental evidence and mediated by histone deacetylase 1 (HDAC1). These interactions lead to predict that a gain of an extra $R B B P 7$ allele should functionally equate a loss in a WHSC1 allele in reference to

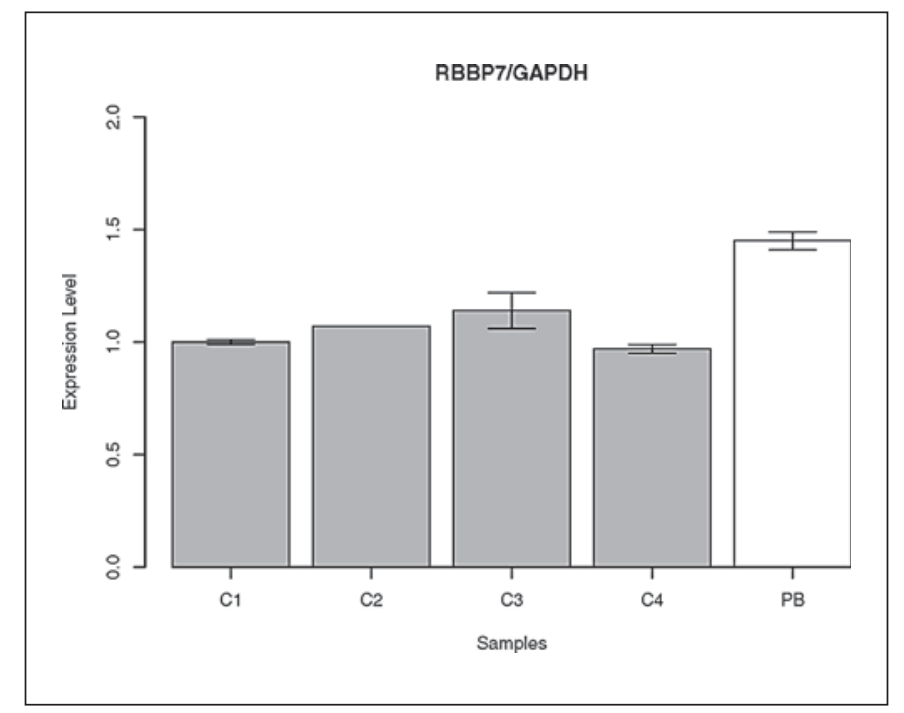

Fig. 3. Relative level of $R B B P 7$ transcript in leukocytes of the proband (PB), compared to 4 unaffected controls (C1-C4). The expected $\Delta \Delta$ Ct ratio is $0.5,1$, and 1.5 when 1,2 or 3 allele copies are expressed, respectively.

HDAC1 activity. RT-qPCR expression analysis shows increased $R B B P 7$ mRNA levels, consistent with the presence of the genomic duplication in the proband's leukocytes ( $\mathrm{p}$ values range from $2.5 \times 10^{-5}$ to $1.9 \times 10^{-08}$ in different control individuals; fig. 3). Instead, no differences in HDAC1 and WHSC1 mRNA levels were detected between the proband and controls (data not shown), confirming the posttranscriptional nature of these interactions. Cellular and animal models will be necessary to ultimately address the functional roles of RBBP7 in clinical conditions sharing dysmorphic features resembling WHS during infancy.

The potential clinical relevance of the extended chromosomal alteration in our patient is supported by the numerous disease genes found within the duplicated Xp22.33p22.12 region. As previously mentioned, duplications of the Xp chromosome have been associated with neurodevelopmental disorders [Honda et al., 2010; Salaria et al., 2012; see DECIPHER website]. Due to the 'Lyon hypothesis', females with X chromosome duplications are generally less severely affected than males [Matsuo et al., 1999]. However, females with a defect in the X chromosome inactivation mechanism show severe mental retardation, developmental delay, dysmorphic facial features, and heart defects [Migeon et al., 1993]. These females somehow resemble our patient. Due to the large size of the Xp duplication in our case, it is difficult to assign a causal role to a specific gene or set of genes. Nonetheless, 
since the present Xp22.33p22.12 duplication harbors more than 100 genes, overexpression of only some of them may have influenced the clinical phenotype, either directly or through an indirect dysregulation of genes located in other chromosomes, as demonstrated for Down syndrome [revised in Lintas et al., 2012]. These scenarios will have to be explored using genome-wide transcriptomic analysis.

\section{Acknowledgments}

We thank the patient and his family for their cooperation. This work was funded by the Italian Ministry of Health (RFPS-2007-5640174 and CCM program 2012), the Fondazione Gaetano e Ma- falda Luce (Milan, Italy), and the Innovative Medicines Initiative Joint Undertaking (EU-AIMS, 115300).

\section{Statement of Ethics}

Written informed consent was obtained from the patient's parents for publication of this case report and any accompanying images. The authors have no ethical conflicts to disclose.

\section{Disclosure Statement}

The authors have no conflict of interest to declare.

\section{References}

Franceschini A, Szklarczyk D, Frankild S, Kuhn M, Simonovic M, et al: STRING v9.1: proteinprotein interaction networks, with increased coverage and integration. Nucleic Acids Res 41(Database issue):D808-D815 (2013).

-Honda S, Hayashi S, Imoto I, Toyama J, Okazawa $\mathrm{H}$, et al: Copy-number variations on the $\mathrm{X}$ chromosome in Japanese patients with mental retardation detected by array-based comparative genomic hybridization analysis. J Hum Genet 55:590-599 (2010).

Lintas C, Sacco R, Persico AM: Genome-wide expression studies in autism spectrum disorder, Rett syndrome, and Down syndrome. Neurobiol Dis 45:57-68 (2012).

MacDonald JR, Ziman R, Yuen RK, Feuk L, Scherer SW: The Database of Genomic Variants: a curated collection of structural variation in the human genome. Nucleic Acids Res 42:D986-D992 (2013).
Matsuo M, Muroya K, Kosaki K, Ishii T, Fukushima $Y$, et al: Random X-inactivation in a girl with duplication Xp11.21-p21.3: report of a patient and review of the literature. Am J Med Genet 86:44-50 (1999).

Migeon BR, Luo S, Stasiowski BA, Jani M, Axelman J, et al: Deficient transcription of XIST from tiny ring $\mathrm{X}$ chromosomes in females with severe phenotypes. Proc Natl Acad Sci USA 90:12025-12029 (1993).

Moretti P, Ferrari M, Di Battista S, Di Battista C: The $4 \mathrm{P}$-syndrome. Case description and literature review. Minerva Pediatr 53:23-28 (2001).

-Piton A, Gauthier J, Hamdan FF, Lafrenière RG, Yang Y, et al: Systematic resequencing of Xchromosome synaptic genes in autism spectrum disorder and schizophrenia. Mol Psychiatry $16: 867-880$ (2011).

Prasad A, Merico D, Thiruvahindrapuram B, Wei J, Lionel AC, et al: A discovery resource of rare copy number variations in individuals with autism spectrum disorder. G3 (Bethesda) 2: 1665-1685 (2012).
Rauch A, Wieczorek D, Graf E, Wieland T, Endele $\mathrm{S}$, et al: Range of genetic mutations associated with severe non-syndromic sporadic intellectual disability: an exome sequencing study. Lancet 380:1674-1682 (2012).

Ropers HH, Hamel BC: X-linked mental retardation. Nat Rev Genet 6:46-57 (2005).

Salaria M, Burgess T, Setyapranata S, Winship I: Phenotype in novel Xp duplication. Am J Med Genet A 158A:2342-2346 (2012).

Wu L, Liu J, Lv W, Wen J, Xia Y, Liang D: An Xp21.3p11.4 duplication observed in a boy with intellectual deficiency and speech delay and his asymptomatic mother. Birth Defects Res A Clin Mol Teratol 97:467-470 (2013).

Zollino M, Bova R, Neri G: From Pitt-RogersDanks syndrome to Wolf-Hirschhorn syndrome and back? Am J Med Genet 66:113115 (1996). 